\title{
EL DERECHO A LA AUTODETERMInACIÓn DE LOS PUEBLOS EN LA PROTODIPLOMACIA PARLAMERTARIA CATALANA: LOS CASOS PALESTINO Y SAHARAUI
}

\section{The right to self-determination of the peoples in the Catalan parliamentary protodiplomacy: the Palestinian and Saharaui cases}

\author{
Raquel Alemañ Navalón \\ Analista. EuropaNova \\ E-mail: raquelalemanybiar@gmail.com \\ Stelios Stavridis \\ Investigador Senior. Araid. Universidad de Zaragoza \\ E-mail: dr.stelios.stavridis@gmail.com
}

1 Autores

\begin{abstract}
Este estudio aborda la paradiplomacia parlamentaria catalana a través del concepto del derecho de autodeterminación. Sin embargo, no se trata de la autodeterminación de Cataluña o incluso de los reclamos de independencia. Se trata de cómo se discute este concepto en el Parlament (parlamento regional) en relación con otros reclamos de ese tipo, a saber, aquellos en el Sáhara Occidental y en Palestina. Por lo tanto, el documento comienza con una discusión sobre conceptos y definiciones. A continuación, se analizará la paradiplomacia catalana en general y, en particular, su protodiplomacia parlamentaria a nivel de la Generalitat (gobierno regional) y también en su Parlament. La siguiente parte evalúa en detalle cómo se han discutido las cuestiones del Sáhara Occidental y de Palestina en el Parlament dentro del contexto más amplio de esta protodiplomacia. El estudio muestra que existen discrepancias entre los partidos políticos a nivel catalán sobre cómo y si instrumentalizan la noción, el concepto y el derecho a la autodeterminación en otros movimientos de independencia frente al propio debate de Cataluña sobre su futuro. Y hay una conclusión.
\end{abstract}

Paradiplomacia parlamentaria; protodiplomacia; autodeterminación de los pueblos; Parlament de Cataluña; partidos políticos; Palestina; Sáhara Occidental.

Key words

Parliamentary paradiplomacy; protodiplomacy; self-determination of the peoples; Parlament of $\mathrm{Ca}$ talonia; political parties; Palestine; Western Sahara. 
This study addresses the Catalan parliamentary paradiplomacy through the concept of the right to self-determination. However, it is not about the self-determination of Catalonia nor the claims of independence. This is about how this concept is discussed in the Parlament (regional parliament) in relation to other claims of that kind, namely, those in Western Sahara and in Palestine. Therefore, the document begins with a discussion about concepts and definitions. Next, we will analyse the Catalan paradiplomacy in general and, in particular, its parliamentary protodiplomacy at the level of the Generalitat (regional government) and also in its Parlament. The following part evaluates in detail how the issues of Western Sahara and of Palestine have been discussed in the Parlament within the broader context of this proto-diplomacy. The study shows that there are discrepancies among the political parties at the Catalan level on how and if they instrumentalize the notion, the concept and the right to self-determination in other independence movements along Catalonia's own debate about its future. And there is a conclusion.

\section{Introducción: ¿por qué es un tema importante?}

Aunque este trabajo aborda el derecho a la autodeterminación y la paradiplomacia catalana, no se tratará la actual crisis de independencia que tanto Cataluña como España están experimentando hoy en día. Además, hemos decidido finalizar el periodo de estudio en 2015 2016, evitando así la "contaminación” de la situación actual. Esto también se hace con la idea de que los desarrollos posteriores a las elecciones del 21 de diciembre de 2017 en Cataluña no afecten fundamentalmente este análisis. Sin embargo, otros sucesos recientes también afectan este estudio, como por ejemplo: el 25 de septiembre de 2017, el Kurdistán iraquí celebró un referéndum por su independencia y desde entonces el proceso está paralizado. Mientras que la opción de referéndum está estancada por el momento en el caso saharaui. Y en el caso palestino, varios representantes internacionales como la Alto Representante de la PESC, la señora Mogherini, en el año 2014, o el presidente estadounidense Donald Trump, en 2017, han pedido una solución a la cuestión palestina.

En este contexto, el paper se plantea cuestiones de investigación específicas: ¿utiliza, y de qué manera, el Parlament otras reclamaciones a la autodeterminación para promover aspiraciones a la independencia de Cataluña? ¿Hay diferencias entre partidos políticos sobre este tema? ¿Hay diferencias entre el Sáhara Occidental y Palestina? ¿Qué tipo de protodiplomacia parlamentaria podemos encontrar en Cataluña?

Este estudio se estructura en seis partes. Empieza con una discusión sobre conceptos y definiciones (Parte 2). A continuación, se analiza la paradiplomacia catalana en general y, en particular, su protodiplomacia parlamentaria a nivel de la Generalitat (gobierno regional) y también en su Parlament (Parte 3). La siguiente parte evalúa en detalle cómo se han discutido las cuestiones del Sáhara Occidental y de Palestina en el Parlament dentro del contexto más amplio de esta protodiplomacia (Parte 4). El estudio muestra que existen discrepancias entre los partidos políticos a nivel catalán sobre cómo y si instrumentalizan la noción, el concepto y el reclamo de la autodeterminación en otros movimientos de independencia frente al propio debate de Cataluña sobre su futuro (Parte 5). Las conclusiones resumen los resultados de este trabajo y apuntan a áreas de posible futura investigación (Parte 6). 


\section{Conceptos, contextos y definiciones}

\subsection{El derecho a la autodeterminación}

Desde el punto de vista de la Carta de las Naciones Unidas tan solo se reconoce el derecho a la autodeterminación en el caso en el que haya habido una descolonización. Hoy en día, existen otros diversos casos de autodeterminación, como podría ser Kosovo (2008) o Sudan del Sur (2011). Desde 1990, tras la disolución de la URSS, en particular, cerca de 15 nuevos estados han sido creados.

Más recientemente desde principio de los años 1990, a partir del desbloqueo de la Unión Soviética y de Yugoslavia, una plétora de nuevos estados ha aparecido en la escena internacional, empezando por los Estados Bálticos en 1991, los estados de la ex-Yugoslavia, como Eslovenia, Croacia, Bosnia-Herzegovina, Montenegro, FYROM y especialmente la región autónoma de Kosovo. En el caso de Kosovo, solo 5 de los 28 Estados miembros no reconocen su independencia, incluyendo España donde, por el contrario, los partidos independientes piden su reconocimiento. Por otro lado, el último Estado "recién llegado", tiene su origen en África, siendo la República de Sudán del Sur que obtuvo su independencia de Sudán en 2011, lo que demuestra que dicho desarrollo no se limita a Europa.

El derecho internacional reconoce el derecho de los pueblos a decidir o derecho a la libre determinación. Los artículos $1 \S 2$ y 55 de la Carta de las Naciones Unidas, establecen dicha regla con el objetivo de "fomentar entre las relaciones de amistad basadas en el respeto al principio de la igualdad de derechos y al de la libre determinación de los pueblos [...]”. Del mismo modo, el 14 de diciembre de 1960, la Resolución 1514 (XV) de la Asamblea General sobre la concesión de la independencia de los países y pueblos coloniales, señaló que "todos los pueblos tienen un derecho inalienable a la plena libertad, al ejercicio de su soberanía y a la integridad de su territorio nacional", así como que "todos los pueblos tienen el derecho de libre determinación; en virtud de este derecho, determinan libremente su condición política y persiguen libremente su desarrollo, económico, social y cultural [...] todo intento encaminado a quebrantar total o parcialmente la unidad nacional y la integridad territorial de un país es incompatible con los propósitos y principios de la Carta de las Naciones Unidas”. Y, complementándose con la Resolución 2625 (XXV)² del 24 de octubre de 1970, confirmará el "principio de la igualdad de derechos y de la libre determinación de los pueblos".

Hoy en día, tanto el territorio del Sáhara Occidental como Palestina, continúan los procesos políticos por obtener su autodeterminación. En el caso del Sáhara, según la Resolución 1452 (XV) de la Asamblea General de la ONU del 15 de diciembre de 1960, sería inscrito sobre la lista de Territorios No Autónomos/TNA ${ }^{3}$ y por consecuencia, en la lista de espera para ser descolonizado. A partir de 1964, la Asamblea General va a llamar a la organización de un referéndum con el fin de obtener el derecho a la libre autodeterminación. Sin embargo, hoy en día, la cuestión del Sáhara Occidental continúa en la agenda de la IV Comisión de la Descolonización de los años 1970.

1 Resolución 1514 (XV), de la Asamblea General sobre la concesión de la independencia de los países y pueblos coloniales, del 14 de diciembre de 1960 http://www.un.org/es/decolonization/declaration.shtml

2 Declaración sobre los principios de Derecho Internacional referente a las relaciones de amistad y a la cooperación entre los Estados de conformidad con la Carta de las Naciones Unidas, Resolución 2625 de la ONU, 24 de octubre de 1970, http://www.un.org/es/comun/docs/?symbol=A/RES/2625(XXV)\&Lang=S\&Area=RESOLUTION

3 Territorios no Autónomos (TNA) de la ONU, http://www.un.org/es/decolonization/nonselfgovterritories.shtml
Desde el punto de vista de la Carta de las naciones Unidas tan solo se reconoce el derecho a la autodeterminación en el caso en el que haya habido una descolonización 
Por otro lado, Palestina ha alcanzado el reconocimiento de su derecho a la libre determinación. Diversas resoluciones de la Asamblea General, del Consejo de Seguridad de las Naciones Unidas, así como otras organizaciones internacionales reconocen el derecho a la libre determinación del pueblo palestino, apoyando incluso la formación y el reconocimiento de un Estado palestino independiente ${ }^{4}$. En 2011, Palestina se convierte en miembro pleno de la UNESCO y obtiene en 2012 la condición de Estado observador no miembro de la Asamblea General de las Naciones Unidas. Recientemente, en septiembre de 2017 se ha incorporado a la Interpol como país miembro.

Varios parlamentos europeos, incluso el Parlamento Europeo, pero también el del único estado miembro de la UE que ha reconocido Palestina hasta la fecha-Suecia (Oliveira Martins, 2015)-, han aprobado resoluciones o declaraciones pidiendo a sus gobiernos el pronto reconocimiento de Palestina como estado independiente (Stavridis, Gianniou, \& Cofelice, 2016).

\subsection{Diplomacia, paradiplomacia, protodiplomacia y diplomacia parlamentaria}

El concepto y la práctica de la diplomacia (Watson, 1982) ha evolucionado mucho en los últimos años: al papel tradicional de un diplomático (comunicación, reportaje, análisis de políticas, negociación y representación), ahora hay nuevas tareas más complejas (por ejemplo, gobernanza, cambio climático o terrorismo).

Además, han aparecido nuevos actores internacionales no estatales (Reinalda, 2011; Hocking, 2011). Como resultado, han surgido nuevos tipos de diplomacia: diplomacia económica (Bayne \& Woolcock, 2011), diplomacia cultural (Kitsou, 2013), diplomacia pública (Melissen, 2005), paradiplomacia (ver abajo), diplomacia de las celebridades (Huliaras \& Tzifakis, 2010), diplomacia deportiva (The Hague Journal of Diplomacy, 2013) y diplomacia parlamentaria (Stavridis \& Jancic, 2017).

También existe una amplia literatura sobre paradiplomacia en general (Aldecoa Luzárraga \& Keating, 1999; Wolff, 2007; Cornago, 2010; Pasquier, 2012; Skoutaris, 2012; Palermo \& Kössler, 2017) y sobre estudios de casos específicos (sobre todo, sobre las relaciones exteriores de Quebec, Escocia, Flandes y Cataluña: ver, inter alia, Noferini, 2016; McHugh, 2015; Criekemans, 2010; Paquin, 2004). Igualmente, existe una clara distinción entre la paradiplomacia en general y la "protodiplomacia" en particular. Esta última se relaciona con la búsqueda de conseguir un estado independiente por parte de un pueblo o de un ente subestatal (McHugh, 2015).

Sin embargo, hay pocos estudios académicos sobre el papel internacional de los parlamentos regionales (subestatales) -conocido como paradiplomacia parlamentaria-. Pero sí que hay alguna literatura sobre sus relaciones con la Unión Europea (Palomares Amat, 2011; Bursens \& Högenauer, 2017).

La diplomacia parlamentaria se ha definido en una literatura emergente como:

La gama completa de actividades internacionales emprendidas por los parlamentarios para aumentar el entendimiento mutuo entre los países, ayudarse mutuamente en mejorar el

4 Serbeto, E. (2014). El Parlamento Europeo apoya el reconocimiento del Estado Palestino. ABC Internacional [en línea]. Disponible en http://www.abc.es/internacional/20141217/abci-estado-palestino-201412171056.html [Consultado el 20/06/2017].

\section{El concepto y la práctica de la diplomacia ha evolucionado mucho en los últimos años}


control de los gobiernos y la representación de los ciudadanos y aumentar la legitimidad democrática de las instituciones intergubernamentales. (Weisglas \& de Boer, 2007)

La totalidad de las actividades que lleva a cabo cada parlamento nacional, tanto como entidad colectiva como iniciativas de parlamentarios individuales, en sus propias relaciones con otros parlamentos nacionales o con los llamados "parlamentos internacionales. (Piazzi, 2015)

O, más sencillamente, como: "Cualquier actividad o acto de carácter diplomático que implica al menos un actor parlamentario (persona o entidad)” (Stavridis, 2016).

Además, debido a la emergencia de nuevas formas de (inter)regionalismo y gobernanza global (Warleigh-Lack, Robinson, \& Rosamond, 2011 ; Telò, Fawcett, \& Ponjaert, 2015), ahora mismo existen esfuerzos de intento de legitimización y democratización (Sabic, 2008): esto ha llevado a una proliferación de instituciones parlamentarias internacionales (IPI) en todo el mundo (Costa, Dri, \& Stavridis, 2013; Kissling, 2011; De Puig, 2008 -ver también Cofelice, 2012-). Esta dimensión no se desarrollará aquí, pero sigue siendo importante de todos modos debido a la existencia en la actualidad de un sistema parlamentario multinivel en muchas macro regiones del mundo, especialmente en Europa (Bursens \& Högenauer, 2017; Jancic, 2015; Crum \& Fossum, 2013). En el caso de Cataluña, es interesante su participación en la AP de la Francofonía (Palomores Amat, 2016).

El Mediterráneo no es una excepción ya que hoy en día existe una escena compleja que trae junto a ella entes subestatales, estados, cuerpos e instituciones parlamentarias regionales, interregionales y globales (Cofelice \& Stavridis, 2017). Así, confirmando no solo, tal como ha sido ya mencionado, la existencia del campo parlamentario multinivel, pero también la plétora de las prácticas actuales de la diplomacia parlamentaria en el Mare Nostrum (Stavridis \& Gianniou, 2016), que incluyen los de los órganos parlamentarios subestatales, es decir, la paradiplomacia parlamentaria como se señaló anteriormente.

\section{El caso de la Generalitat: de paradiplomacia a protodiplomacia}

La literatura sobre la paradiplomacia de las comunidades autónomas es muy amplia, en particular desde las reformas de 2006 (García Pérez, 2009). Se ha prestado especial atención a las denominadas "regiones históricas" de España; algunas de ellas con fuertes movimientos independentistas, en particular el País Vasco (de Castro Ruano y Ugalde Zubiri, 2004; 2011) y Cataluña. Este estudio no se focaliza en los pros y contras de la independencia catalana, tampoco en sus relaciones generales con y en el interior de la UE (Noferini, 2016), ni sobre qué objetivos han sido promovidos en el actual gobierno catalán y partidos políticos afines a este objetivo en España, en Europa y más allá. Este trabajo se centrará en Cataluña, pero primero algunos puntos generales introductorios sobre la paradiplomacia catalana y la protodiplomacia.

La identidad, la cultura y la lengua catalana, así como su fuerte influencia económica dentro de España, son factores que han favorecido un acercamiento por parte del gobierno catalán hacia Europa, sobre todo en cuanto a la cuestión regional y a otras estructuras tales como la Asamblea de las Regiones Europeas o el Comité de las Regiones. No obstante, esta política marcada por el afán de desarrollar relaciones con sus vecinos más próximos para hacerse hueco en la esfera euromediterránea, ha condicionado la agenda de la política exterior de España.

\section{La literatura}

sobre la

paradiplomacia de las comunidades

autónomas es

muy amplia, en

particular desde las reformas de 2006 
Un reciente artículo de Laura Feliu y Francesc Serra sobre la diplomacia parlamentaria catalana en el Mediterráneo, confirma que "la región que más interés desata a los diputados catalanes es, sin duda, el Medio Oriente y el Norte de África” (Feliu y Serra, 2016, p. 130). También, sostienen que inicialmente se hizo hincapié en las cuestiones locales, aunque lentamente hubo más de una "acción externa" (este es el término exacto utilizado en la Constitución Española de 1978 para las relaciones exteriores del CC. AA.).

El mismo estudio apunta también a un papel externo más activo durante el Gobierno tripartito (ver abajo), pero se produjo un cambio sustancial y cualitativo con el lanzamiento de la campaña proindependencia en 2003. En el periodo que condujo entre 2006-2010, se observan "muchos más viajes parlamentarios al exterior para discutir la "necesidad de independencia”, aunque este no sea un tema que vayamos a analizar aquí, sino que, como ya se ha dicho con anterioridad, se centra más en cómo se discute en el Parlamento de Cataluña otros movimientos de "independencia" (ceteris paribus) en el mundo.

Las varias presidencias de la Generalitat han sido las siguientes: Pujol (1980-2003), Maragall (2003-2006), Montilla (2006-2010), Mas/Puigdemont (2010-2016/2017).

La proyección exterior de Cataluña con anterioridad a la Guerra Fría era todavía horizontal hasta la llegada de la presidencia de CiU con Jordi Pujol a la cabeza (1980-2003), quien a partir de sus ambiciones conduciría la política catalana hacia la institucionalización y el aumento del volumen de las actividades exteriores. Cataluña se reorganizará a partir del decreto 141/2003 del 10 de junio 2003 bajo tres ejes: "las relaciones territoriales, la coordinación exterior y la cooperación para el desarrollo” (Bizoux, 2006, p. 7).

Entre 2003 y 2006, con Pasqual Maragall (PSC) a la cabeza, la proyección exterior se orientaría más a una estructura vertical (Criekemans, 2010, p. 44). Posteriormente, entre 2006 y 2010 , con José Montilla (PSC), dirigiría el control sobre la delegación catalana ante la UE, con sede en Bruselas, mientras que Josep Lluís Carod-Rovira, desde la vicepresidencia, dirigiría la promoción y la cooperación exterior de la Generalitat. No obstante, será junto a Artur Mas (CiU), entre 2010 y 2016, cuando la ampliación de la administración de Cataluña vendrá con más fuerza, en medio de un contexto marcado por un fuerte movimiento independentista que impulsará la proyección exterior de Cataluña hasta la actualidad junto a Carles Puigdemont.

De hecho, existen diversos tipos de "diplomacia", aunque en este análisis tan solo abordaremos la diplomacia internacional relacionada con el derecho a la autodeterminación de Cataluña con el fin de comprender las acciones llevadas por el Parlamento Catalán. La participación internacional de Cataluña se extiende a lo largo de acuerdos específicos con otras entidades y organizaciones, como por ejemplo, California, la provincia coreana de Kyonggi, Escocia, la Asamblea Nacional de Quebec o el Centro Nacional para la Investigación Científica (Wolff, 2007).

La crisis económica también perjudicaría el presupuesto catalán provocando reducciones presupuestarias que afectarían a la ampliación externa de la política catalana. En diciembre de 2011, durante el mandato de Artur Mas, la delegación en Buenos Aires (Argentina) sería cerrada. Dicha oficina, formaría parte de la red de "embajadas", conocidas así las oficinas del Govern en el extranjero, las cuales tendrían un coste muy elevado ${ }^{5}$. De este modo, continuaría

\section{La proyección} exterior de

Cataluña con anterioridad a la Guerra Fría era todavía horizontal hasta la llegada de la presidencia de cill con Jordi Pujol a la cabeza 
contando con cinco oficinas: París (Francia), Berlín (Alemania), Nueva York (Estados Unidos), Bruselas (Bélgica) y Londres (Reino Unido).

\section{Estudios empíricos del caso del Parlament}

A partir del artículo escrito por Feliu y Serra, encontramos una descripción de la estructura del Parlamento catalán en cuanto a sus relaciones internacionales, al igual que aporta los instrumentos que fueron aprobados por el Parlament, el Consejo de Portavoces y la Oficina del Presidente de la Cámara y que han sido utilizados desde el año 2000 en términos de resoluciones, declaraciones institucionales de contenido internacional (más de cuarenta) y mociones para hacer frente a dichas cuestiones. Entre las declaraciones, aparece una gran concentración en las mismas áreas geográficas y materias, seis declaraciones referentes al terrorismo islámico, cinco al conflicto palestino, cuatro al Sáhara, dos a las revueltas árabes, dos para el conflicto sirio, dos a las crisis de los refugiados, entre otras (Feliu y Serra, 2016, p. 131) ${ }^{6}$.

En líneas generales, el actual Estatuto de Autonomía de Cataluña refleja a través de su preámbulo su compromiso con todos los pueblos en la construcción de "un orden mundial pacífico y justo"

Nuestro análisis empieza en el año 2003, tras el cambio de gobierno con la entrada de Pasqual Maragall. El nuevo gobierno de izquierdas impulsará políticas con un aire más progresista. Además, analizando los debates parlamentarios catalanes, se puede observar como entre los años 2003 y 2006, empiezan a surgir preocupaciones parlamentarias sobre el conflicto del Sáhara Occidental y Palestina, habiendo pasado por un vacío sin indicios de actividad. A partir de este momento se observa un mayor interés en ciertos grupos que conforman el Parlament, de una manera indirecta, de promover el apoyo constante a favor del derecho a la autodeterminación, discurso utilizado a lo largo de estos años por los independentistas. Por esta razón nos centramos en el periodo posterior a 2003.

En 2003, el Parlament se pronunciará sobre la guerra en Iraq y la situación en el Próximo Oriente, en el cual nombrará el conflicto Israel-Palestina ${ }^{8}$. En cuanto al Sáhara Occidental, no hay datos hasta el momento9. Será a partir de 2004, cuando diversos diputados expondrán preguntas directas acerca de diferentes movimientos llevados a cabo en ambos territorios. Durante la legislatura VI, la Comisión de Organización y Administración de la Generalitat y Gobierno Local, adoptaría la Resolución 1699/VI del Parlament de Cataluña, sobre el Plan director de

6 Feliu y Serra (2016) aportan más detalles: a lo largo de estos años se crearán diversos comités legislativos permanentes junto al Comité de Acción Exterior, Unión Europea y Cooperación, conocido actualmente como el Comité de Acción Exterior y Cooperación, Relaciones Institucionales y Transparencia. Otros comités son el Comité de Justicia y Derechos Humanos o la Comisión de Economía, Finanzas y Presupuestos. Otra de las actividades exteriores sería la creación de Grupos Interparlamentarios como el Grupo Interparlamentario para la Paz y la Libertad en el Sáhara en 2006 y, el Grupo Interparlamentario por Palestina más recientemente en 2016. En el caso del grupo creado para el Sáhara, también participarán la delegación en Cataluña del Frente Polisario, la Coordinadora Catalana de Solidaridad por los Consejos Locales con el pueblo saharaui y la Asociación Catalana de Amigos del Pueblos Saharaui, entre otros, junto a los que se han promovido resoluciones parlamentarias y declaraciones. Cataluña también ha realizado intercambios de visitas, aunque en este caso la mayor parte han sido realizados con el Reino de Marruecos.

7 Estatut d'autonomia de Catalunya, p. 20. Edició 2016. Disponible en http://www.parlament.cat/document/cataleg/48089.pdf [Consultado el 14/06/2017).

8 En 2001, ya existe la Resolució 696/VI del Parlament de Catalunya, sobre la fi de la violencia a l’Orient Mitjà entre Israel i el poble palestí.

9 Butlletí Oficial del Parlament de Catalunya, Legislatura VI, Nº 392, 17 febrer 2003. 
cooperación al desarrollo, propuesta por el Govern así como por el PP, CiU, ERC i ICV. Uno de los pilares de la construcción nacional catalana sería, y es, la cooperación internacional.

El Plan parte de la consideración de la cooperación al desarrollo como una dimensión necesaria de la internacionalización de Cataluña. Como dice la exposición de motivos de la Ley 26/2001, de cooperación al desarrollo, "hoy no se puede construir un proyecto nacional creíble ni una sociedad democrática avanzada sin participar activamente i decididamente en la construcción de un orden internacional más justo y solidario” ${ }^{10}$.

Ya, en dicho pla, la concepción de la cooperación es y será uno de los valores que integran la identidad catalana es "el derecho de los pueblos a la defensa y la promoción de la cultura, la lengua y la identidad propias" ${ }^{11}$.

Dicha cooperación desde 2003 hasta 2006, está dirigida a países que consideran desde la Generalitat prioritarios, siendo para ellos, sobre todo, los países y los pueblos del área geográfica del Mediterráneo, especialmente los del Magreb. No obstante, ni en la Resolución 1699/VI ni en la 351/VII se nombra en ningún momento el territorio del Sáhara Occidental ni de Palestina ${ }^{12}$. El Sáhara, finalmente será incluido como país prioritario argumentando su pasado histórico con el Estado español y a consecuencia de la incorporación de un gobierno más progresista a través de la Resolución 219/VII sobre la declaración del Sáhara occidental como zona prioritaria de inversión en el Plan director de cooperación al desarrollo 2003-2006 ${ }^{13}$. A partir de esta, se añadiría también Palestina ${ }^{14}$. A lo largo de 2004, ambos territorios recibirán ayudas según su estatuto de países prioritarios, por lo tanto, en el caso del Sáhara recibirá 108.889,51 euros en 2004, y 252.968,52 euros en 2005, y en el caso de Palestina, 242.000,00 euros en 2004 y $892.784,27$ euros en $2005^{15}$, y así hasta día de hoy.

El cambio de posición que se empezaría a gestar en 2004, en el Parlament hacia una vertiente más próxima al derecho de los pueblos a decidir, dará lugar a nuevas decisiones y debates con respecto al Sáhara Occidental y Palestina.

En este mismo año, el Parlament aprobaría la Resolución 99/VII del Parlament català sobre el apoyo al pueblo saharaui y a las resoluciones y el plan de paz del Consejo de Seguridad de las
El cambio de posición que se empezaría a gestar en 2004, hacia una vertiente más próxima al derecho de los pueblos a decidir, dará lugar a nuevas decisiones y debates con respecto al Sáhara Occidental y Palestina

10 Res 1699/VI del Parlament de Catalunya, sobre el Pla director de cooperació al desenvolupament, Tram. 25900001/06, Sessió num. 31, 19.02.2003, DSPC-C 428, BOPC 401.

11 Res 1699/VI Op.cit., Res 66/VIII, Res 754/VIII.

12 Aunque durante este periodo no se proponga como países prioritarios a Palestina y el Sáhara Occidental, sí que se observa la preocupación de diversos diputados de diversos partidos a través de las preguntas realizadas en los debates del Parlamento y que constan en los Boletines Oficiales, como Josép M. Pelegri i Aixut (CiU), Elena Ribera i Garijo (CiU), Felip Puig i Godes (CiU), Marta Llorens i García (CiU), Rafael López i Rueda (PP), Daniel Sirera i Bellés (PP), Consol Prados Martínez (Socialistes-Ciutadans pel Canvi), Josefina Cambra i Giné (CiU).

13 Butlletí Oficial del Parlament, Legislatura VII, No 143,7 de febrero de 2005; No 175 , 25 d’abril de 2005. La última revisión dio lugar a la Resolució 351/VII del Parlament de Catalunya, sobre la revisió del Pla director de cooperación al desenvolupament 2003-2006, de l'l de febrero de 2006; Res 219/VII del Parlament de Catalunya, sobre la declaración del Sáhara Occidental com a zona prioritaria d'actuació en el Pla director de cooperación 2003-2006, Tram 250-00316/07, Sessió núm. 11.23.04.3005, DSPC-C 174, BOPC 175.

14 Butlletí Oficial del Parlament, Legislatura VII, No 241, 14 de noviembre de 2005.

15 Butlletí Oficial del Parlament, Legislatura VIII, No 68, 10 de maig de 2007. 
Naciones Unidas ${ }^{16}$, propuesta por el grupo parlamentario del Partit Popular de Catalunya ${ }^{17}$. Además, con la adopción de la Resolución 102/VII del Parlament de Catalunya, sobre la orientación política general del Consejo Ejecutivo, el Parlament pedirá apoyo para "la liberación o el reconocimiento de los pueblos como el amazic y el saharaui" y "el pleno desarrollo de las libertades nacionales del pueblo palestino" ${ }^{18}$.

Siguiendo por la línea de la proclamación de las naciones sin Estado, remontándonos a la celebración de la Cumbre por la Declaración de Barcelona firmada en 1995, Cataluña promoverá en 2006 la sensibilización "a los Països Catalans sobre la realidad de las naciones sin Estado de todo el Mediterráneo”. No obstante, a la hora de nombrar a los países receptores de financiación, nombran la Cabilia junto a Argelia entre paréntesis y del mismo modo pasa con el Sáhara Occidental, junto a Marruecos. Por el contrario, Palestina aparece individualmente y, además, señalan Marruecos ${ }^{19}$ y Palestina como países prioritarios de la cooperación catalana ${ }^{20}$. Esto quiere decir que, se considera por una parte Palestina como país, mientras que el Sáhara todavía no se le da este estatuto. El mismo caso ocurre cuando observamos que en los Procesos de Barcelona, Palestina participa como país mientras que no hay datos sobre el Sáhara, al igual que en la realización de la Asamblea de la Red Euromediterránea por los Derechos Humanos.

A partir del año 2007 con la Resolución 66/VIII21 y posteriormente en la Resolución 754/VIII22 (2010), el Sáhara aparecerá en las prioridades geográficas en el Plan Director de Cooperación al Desarrollo. No obstante, en la resolución de 2010, en cuanto a los valores referentes a los derechos de los pueblos, se expone que "algunos de los socios de la cooperación al desarrollo de la Generalidad son pueblos sin Estado. Este es el caso de pueblos como los amazics o los kurdos de la región mediterránea o los pueblos aimara y quitxua de la región andina, o los diversos pueblos de la región mesoamericana y la región de África occidental, entre otros"; sin nombrar en ningún caso el territorio saharaui ocupado por Marruecos. Posteriormente, Palestina será expuesto como país prioritario mientras que, el Sáhara Occidental será señalado como país o territorio preferente ${ }^{23}$. Este cambio de identificación de zona prioritaria a territorio preferente sería cuestionada por Rafael López i Rueda, del Grup Parlamentari del PPC, quién preguntaría: “¿Cuál es, según el Govern de la Generalitat, la causa de la exclusión del Sáhara Occidental como ‘zona prioritaria de actuación’ del Plan Director de Cooperación al Desarrollo

16 Butlletí Oficial del Parlament de Catalunya, Legislatura VII, Nº6, 19 de juliol de 2004; Resolució 99/VII del Parlament de Catalunya, sobre el suport al poble sahrauí i a les resolucions i el pla de pau del Consell de Seguretat de les Nacions Unides, 1 de juliol de 2004.

17 Butlletí Oficial, Lg. VII, Nº55, 17 de maig de 2004; Butlletí Oficial, Lg. VII, Nº59, 24 de maig de 2004; Butlletí Oficial, Lg. VII, Nº6, 7 de juny de 2004; Butlletí Oficial, Lg. VII, Nº6, 11 de juny de 2004; Butlletí Oficial, Lg. VII, Nº6, 19 juliol de 2004. También, Res 99/VII del Parlament Catalá sobre el suport al poble sahrauí i a les resolucions i el pla de pau del Consell de Seguretat de les Nacions Unides.

18 Res102/VII del Parlament de Catalunya, sobre l'orientació política general del Consell Executiu. Tram 25000284/08, Sessió núm. 12.13.12.2007, DSPC-C 218, BOPC 188.

19 Butlletí Oficial del Parlament de Catalunya, Legislatura VII, N²81, 25 de gener de 2006.

20 Butlletí Oficial del Parlament de Catalunya, Legislatura VII, N²81, 25 de gener de 2006.

21 Res 66/VIII del Parlament de Catalunya, sobre el Plan director de cooperación al desenvolupament 2007-2010, Núm. exp. 259-00001/08.

22 Res 754/VIII del Parlament de Catalunya, per la qual s'aprova el Pla director de cooperación al desenvolupament 2011-2014, Núm. exp.: 259-00003/08, 22 de juliol de 2010.

23 Butlletí Oficial del Parlament, Legislatura VIII, No 78, 5 d e juny de 2007; Legislatura Ven el III, Nº 112 , 17 de juliol de 2007.

\section{Cataluña}

promoverá

en 2006 la sensibilización "a los Països Catalans sobre la realidad de las naciones sin Estado de todo el Mediterráneo" 
2007-2010?”24. Finalmente, el Sáhara sería establecido como país y territorio prioritario ${ }^{25}$, junto a Palestina. Este giro, podría ser consecuencia de la firma de un programa sobre emigración sin presencia de la embajada de España, conocido como Programa Catalunya-Magrib ${ }^{26}$. También, se abrió la Oficina de la Agencia Catalana de Cooperación al Desarrollo en Marruecos, y Cataluña participaría en Nador, en un encuentro internacional sobre Federalismos y autonomías dentro del Mundo y en Marruecos ${ }^{27}$.

En 2015, Palestina y el Sáhara volverían a aparecer en la Resolución 1030/X como territorio prioritario, como consecuencia de los procesos independentistas que se han acontecido en esta región a lo largo de estos años. A partir de esta resolución afirmarían que Cataluña vela por "el respeto al derecho de los pueblos a decidir su futuro libremente" y "al derecho de los pueblos a decidir democráticamente el propio futuro por medio de la participación”.

Finalmente, hay que tener en cuenta que desde 2005 hasta hoy en día, ha habido nueve resoluciones directamente tratando del Sáhara occidental y desde 2011, cuatro directamente de Palestina.

\subsection{Ejemplos específicos de decisiones y acciones del Parlament (I): Sahara Occidental, un primer caso de apoyo a la protodiplomacia de la Generalitat a través del caso saharaui}

Como ya se ha nombrado anteriormente, en el año 2003, el Sáhara sería introducido como país prioritario en el Plan Director de Cooperación. En 2004, tras una entrevista realizada con el representante del Frente Polisario, Emborik Ahmed, el Govern se pronunciarían acerca del derecho de autodeterminación saharaui en el Parlament, contribuyendo en este caso al incremento de las ayudas para los campamentos saharauis ${ }^{28}$ :

Como ya se ha puesto de manifiesto, el Govern tiene interés en conocer de primera mano la situación del proceso de paz para el Sáhara Occidental que impulsa la ONU y en la cual el Frente Polisario es parte implicada, y apoya la búsqueda de soluciones negociadas que, en el marco de esta organización internacional y en cumplimiento de las resoluciones adoptadas en su sí, garantizan el derecho a la autodeterminación del pueblo saharaui $[. .$.$] así como considera que se debe realizar todos los esfuerzos que$ sean necesarios para alcanzar esta finalidad ${ }^{29}$.

Como consecuencia de los hechos acontecidos en los territorios ocupados del Sáhara Occidental en 2005 (represión de manifestaciones pacíficas y violaciones de los derechos humanos en

24 Butlletí Oficial del Parlament, Legislatura VIII, No84, 12 de juny de 2007. (Pregunta retirada posteriormente en: Butlletí Oficial, Legislatura VIII, N N 124, 12 de septembre de 2007 o en Diari de Sessions del Parlament de Catalunya, Legislatura VII, Série C-Nº 145, 26 de juliol de 2007).

25 Butlletí Oficial del Parlament, Legislatura VIII, No 115, 23 de juliol de 2007.

26 “Signat un conveni que promourà la integració dels menors marroquins en tots els àmbits”, Generalitat de Catalunya [en línea], Juny de 2007. Disponible en: http://www.gencat.cat/acordsdegovern/20070605/02.htm [Consultado el 28/06/2017]

27 Butlletí Oficial del Parlament, Legislatura VIII, No 331, 14 d'octubre de 2008.

28 Adjudicación de 950.000 euros "en cultura de la pau i països en conflicto i post-conflicte com Palestina, el Sàhara Occidental, Colòmbia i Bòsnia-Herzegovina”. Butlletí Oficial del Parlament, Legislatura VII, Nº 128,20 de desembre de 2004.

29 Butlletí Oficial del Parlament de Catalunya, Legislatura VII, Nº41, 6 d'abril de 2004, p. 9.

\section{El Govern tiene} interés en conocer de primera mano la situación del proceso de paz para el Sáhara Occidental que impulsa la ONU y en la cual el Frente Polisario es parte implicada 
Gdeim Izik), el Parlament de Catalunya declaró: "reitera las diversas declaraciones que se han hecho los últimos años en pro del derecho a la autodeterminación del pueblo saharaui [...] de la celebración del referéndum que prevé el Plan Baker y de la suficiencia de la ayuda humanitaria para los campamentos $[\ldots]]^{30}$.

Por otro lado, en 2007, con motivo del Año Internacional de Solidaridad con el pueblo Saharaui, el Parlament de Cataluña realizaría una declaración en la que volvería a pedir la libre determinación del pueblo del Sáhara Occidental, así como la realización de un referéndum y soluciones para su situación ${ }^{31}$.

A partir de 2008, desde la Generalitat, se incrementaría la financiación de proyectos destinados a incrementar las actividades relativas a la educación y sensibilización de la sociedad catalana ante la cooperación y la solidaridad internacional. En este caso, una de las entidades financiadas sería Diomira Barcelona junto a su campaña de sensibilización de la población catalana sobre la necesidad de ayudar al pueblo saharaui. También, se proporcionaría ayuda a la Associació Famílies Solidàries Nens Saharauis de Hospitalet de Llobregat, con la donación de vehículos para el transporte urgente entre wilayas y la mejora de las condiciones; para la Fundación Lleida Solidària con la mejora de las condiciones e infraestructuras de los campos de refugiados.

Entre 2008 y 2010, se observan nuevas cuestiones planteadas por parte de los parlamentarios referentes a visitas de personalidades saharauis y marroquíes para que expliquen la situación del Sahara. Debemos remarcar el convenio de colaboración ${ }^{32}$ firmado entre Cataluña y el Frente Polisario con el objetivo de atender las necesidades de la población saharaui con proyectos de cooperación, de asistencia humanitaria y de sensibilización ${ }^{33}$. En 2009, además, durante la huelga de hambre de la activista saharaui Aminatu Haidar, el Parlament redactaría una declaración sobre su situación, denunciando la violación de los derechos humanos e instando al Gobierno del Estado español a tomar medidas frente a dicha cuestión ${ }^{34}$. Los derechos humanos junto a este caso darían lugar a una propuesta directa por parte del Intergrup de la Pau i la Llibertat al Sáhara de una resolución sobre la violación de los derechos humanos del pueblo saharaui, que se cristalizaría con la Resolución 724/VIII del Parlament de Catalunya, sobre el respeto de los derechos humanos y las libertades de la población del Sáhara Occidental ${ }^{35}$.

Entre 2011 y 2012, surgirán diversas propuestas de resolución con referencia a los derechos humanos, así como al derecho de la autodeterminación. Finalmente, se aprobarían la Resolución 60/IX sobre la situación del Sáhara en la que "reitera [...] el derecho a la autodeterminación del pueblo saharaui, y también del cumplimiento de la legalidad internacional y de las

30 Declaració institucional del Parlament de Catalunya amb motiu dels darrers esdeveniments al Sáhara Occidental, Palau del Parlament de Catalunya, 30 de juny de 2005.

31 Butlletí oficial del Parlament, Legislatura VIII, Nº174, 3 de desembre de 2007.

32 Existe otro convenio firmado el 21 de abril de 2005, con el objetivo de apoyar las iniciativas a favor de la paz y en busca de una solución justa para la población refugiada.

33 "La Generalitat reforça la seva col-laboració amb el Front Polisario per atendre les necessitats de la població sahrauí", Sala de premsa Generalitat de Catalunya [en línea], 2009. Disponible en http://premsa.gencat.cat/ pres_fsvp/AppJava/notapremsavw/94402/ca/generalitat-reforca-collaboracio-front-polisario-atendre-necessitatspoblacio-sahraui.do [Consultado el 28/06/2017].

34 Declaració de la Junta de Portaveus del Parlament de Catalunya sobre la situació de l'activista sahrauí Aminatu Haidar, Tram. 401-00044/08,9 de desembre de 2009. In Butlletí Oficial del Parlament, Legislatura VIII, Nº593, 9 de desembre de 2009.

35 Res 724/VIII, del Parlament de Catalunya, sobre el respecte dels drets humans i les llibertats de la población del Sàhara Occidental, Tram. 250-02777/08, Sessió núm 27.03.06.2010, DSPC-C 860, BOPC 743.

\section{A partir de} 2008, desde la Generalitat, se incrementaría la financiación de proyectos destinados a incrementar las actividades relativas a la educación y sensibilización de la sociedad catalana

ante la cooperación y la solidaridad internacional 
resoluciones de las Naciones Unidas, incluida la relativa a la celebración del referéndum de autodeterminación”36, y la Resolución 91/X sobre el respecto de los Derechos Humanos i las libertades de la población del Sáhara Occidental, en la que "ratifica la disposición abierta a colaborar para encontrar una solución justa y definitiva [...], que respete el derecho del pueblo saharaui de poder elegir su futuro a través de un referéndum" ${ }^{37}$. Además, sería aprobada la Resolución 162/IX ${ }^{38}$ sobre la Minurso para que asuma la protección de los derechos humanos en el Sáhara, la resolución 605/IX ${ }^{39}$ sobre las gestiones con el Gobierno de España para la formalización de relaciones diplomáticas con el Frente Polisario, único representante del Sáhara, y la resolución 730/IX ${ }^{40}$ en apoyo a las actuaciones de protección de los derechos humanos en dicho territorio.

A partir de 2013, otras resoluciones aparecerán sobre la mesa en referencia al Sáhara ${ }^{41}$, como la Resolución 719/X $\mathrm{X}^{42}$ (2014) de apoyo al Derecho a la Autodeterminación del Sahara Occidental, a partir de la cual insta al Gobierno a "mostrar una actitud decidida y firme en la defensa del derecho del pueblo saharaui a la autodeterminación” ${ }^{3}$. Por otro lado, también propondrán resoluciones relativas a temas de solidaridad como la Resolución 1037/ P4 $^{4015}$ ) y la Resolución 357/XI ${ }^{45}$ (2016), así como la declaración institucional de solidaridad por la catástrofe humanitaria en los campamentos de Tinduf ${ }^{46}$.

En 2014, se observa cierta tensión a partir de la interpelación del Grup Parlamentari d’Iniciativa per Catalunya Verds-Esquerra Unida i Alternativa, publicado en el Boletín del 2 de julio de $2014^{47}$, frente a la Interpelación del Gobierno sobre la política de acción exterior (tram300/00188/10). En esta, se considera que el gobierno de Catalunya ha mantenido una "actitud pasiva, cuando no cómplice", a lo largo de las diversas legislaturas, con gobiernos como el de Marruecos. Con referencia al caso saharaui, el 22 de abril de 2015, el gobierno presentaría el

36 Res 60/IX del Parlament de Catalunya, sobre la situació del Sàhara Occidental. Tram. 250-00174/09, Sessió nú, 3 , 04.05.2011, DSPC-C 71 .

37 Res 91/X del Parlament de Catalunya, sobre el respecte dels drets humans i les llibertats de la població del Sàhara Occidental, Tram. 250-00144/09, Sessió nú, 3. 26/05/2011, DDSPC-C83.

38 Res 160/IX del Parlament de Catalunya, sobre el reforçament de les actuacions de cooperació al desenvolupament i de construcció de la pau amb Palestina, Tram- 250-00257/09, Sessió núm. 4, 01.070201 1, DSPC-C 112.

39 Res 605/IX, del Parlament de Catalunya, sobre les gestions amb el Govern de l'Estat per a la formalització de relacions diplomàtiques amb el Front Polisario, Tram 250-00923/09, Sessió núm. 9, 17.05.2012, DSPC-C 318.

40 Res 730/IX del Parlament de Catalunya, de suport a les actuacions per a la protecció dels drets humans al Sàhara Occidental, Tram. 250-01103/09, Sessió núm. 14, 13.97.2012, DSPC-C 375.

41 Proposta resolució sobre la llibertat desl presos polítics saharauís al Marroc i el garantiment dels drets fondamentals del poble saharauí, finalmente retirada. Butlletí Oficial del Parlament, Legislatura X, N 149, 23 de septembre de 2013.

42 Res 719/X de suport al dret dl'Autodeterminació del Sàhara Occidental, Tram. 250-00991/10, Sessió núm. 15, 20.06.2014, DSPC-C 442.

43 Butlletí oficial del Parlament, Legislatura X, No 101,17 de juny de 2013.

44 Res 1037/X, del Parlament de Catalunya de solidaritat amb el poble sahrauí i de suport al seu dret a l'autodeterminació, Tram. 250-01578/10, Sessió 27, 29.05.2015, DSPC-C 728.

45 Res 357/XI del Parlament de Catalunya, sobre la mort de l'activista Brahim Saïka, Tram. 250-00393/11, Sessió Núm. 13, 03.11.2016, DSPC-C 241.

46 Declaració del Parlament de Catalunya de solidaritat amb els refugiats sahrauís amb motiu de la catàstrofe humanitària als campaments algerians de Tindouf, Tram. 401-00004/1 1, 02 de març de 2016.

47 Butlletí Oficial del Parlament de Catalunya, Legislatura X, No 350, 2 de julio de 2014. 
Pla Director del Marroc ${ }^{48}$, planteando diversas cuestiones dentro del Parlament sobre el respeto de las fronteras del Sáhara durante la legislación de este plan. Además, Catalunya pretendería reforzar el sector empresarial en Marruecos, y se plantea si en un futuro habrá lugar a un Pla Director del Sáhara Occidental "dado que es un territorio preferente, que como Catalunya tiene pendiente la celebración de un referéndum de autodeterminación” ${ }^{4}$.

\subsection{Ejemplos específicos de decisiones y asociaciones del Parlament (II): Palestina, un segundo caso de apoyo a la protodiplomacia de la Generalitat a través del caso palestino}

Como ya se ha explicado con anterioridad, la primera vez que se escuchará hablar de Palestina será en 200350. Del mismo modo, en 2006 y siguiendo con la idea de la Cumbre de 1995, Palestina aparecerá individualmente, con estatuto de país, al contrario que en el caso del Sáhara. Del mismo modo ocurrirá en la realización de la Asamblea de la Red Euromediterránea por los Derechos Humanos ${ }^{51}$.

En cuanto a acciones directas en el territorio, en 2005 una delegación del Parlament Catalán de observadores internacionales sería desplazada a Palestina en vista de las elecciones presidenciales, siendo la primera vez que la Generalitat de Cataluña constituiría una delegación de observadores internacionales al margen del gobierno central de España. Los integrantes de dicha delegación fueron Pere Vilanova, Jaume Saura, Julián Artacho, Jordi Vaquer, Esmeralda Francisco, Tamyko Ysa, Josefina Cambra (CiU), Antoni Comin (PSC-CpC), Pilar Dellunde (ERC), Rafael López (PPC), Dolors Camats (ICV) ${ }^{52}$. Tras las elecciones realizadas en Palestina, Cataluña continuará cooperando para el buen desarrollo y paz en el territorio. Por otro lado, ese mismo año, desde el 19 al 22 de mayo, el presidente de la Generalitat, Pasqual Maragall, visitaría Israel, Palestina y Jordania. El resultado de este evento fue la adquisición de empresas de origen israelí como Maquinaria Canigo, Klein Iberica o Espancid³, entre otras, además de cooperación tecnológica y de investigación en ámbitos de interés común, favoreciendo al desarrollo de la economía catalana.
48 Acuerdo GOV/122/2014, de 15 de septiembre, por el que se aprueba el Plan Marruecos 2014-2017 del Gobierno de la Generalitat de Cataluña, Diari Oficial de la Generalitat de Catalunya, Núm. 6708-17.9.2014. Disponible en http://portaldogc.gencat.cat/utilsEADOP/PDF/6708/1371893.pdf [Consultado el 29/06/2017].

49 Butlletí oficial del Parlament, Legislatura X, Nº602, 16 de juny de 2015.

50 Ver apartado 3.

51 Butlletí Oficial del Parlament de Catalunya, Legislatura VII, No 439, 6 d'abril de 2009; No 486, 15 de juny de 2009.

52 Observadores catalanes asisten a las elecciones palestinas. El País [en línea], 2005. Disponible en: http://elpais.com/diario/2005/01/07/catalunya/1105063643_850215.html [Consultado el 27/97/2017].

53 Butlletí Oficial del Parlament, Legislatura VII, No 206, 5 de juliol de 2005; Legislatura VII, N²07, 5 de juliol de 2005.
En 2005 una delegación del Parlament Catalán sería desplazada a Palestina, siendo la primera vez que la Generalitat constituiría una delegación al margen del gobiemo central de España 
Del mismo que con el Sáhara, en 2007 con la Resolución 66/VIII ${ }^{54}$ y posteriormente en la Resolución 754/VIII55 (2010), Palestina aparecerá en las prioridades geográficas en el Plan Director de Cooperación al Desarrollo. Pero, en la resolución de 2010, en cuanto a los valores referentes a los derechos de los pueblos, no será nombrada Palestina, sometido por Israel.

En 2008, del mismo modo que el Sáhara, Palestina ${ }^{56}$, también se subvencionaría el Servei Civil International-SCI, junto a su programa de formación en periodismo, por un lado, y por otro, tanto en Israel como en Palestina, el encuentro de mujeres catalanas y palestinas de Israel, que subvencionaría estancias de solidaridad en países y pueblos en vías de desarrollo. Por otro lado, otra de las entidades subvencionadas sería Nexes Interculturals de Joves per Europa, en la que estarían países como Siria, Líbano, Jordania, Israel y Palestina, junto a Catalunya en el proyecto Follow the women-Women for peace, y el proyecto AMAL-TIKVA, en el que el principal protagonista para abrir conciencias sería el vídeo social como herramienta de documentación y resistencia pacífica. También, la Xarxa d'enllaç amb Palestina, proyecto en el que se pide un proceso de paz justo para Palestina ${ }^{57}$. En 2009, también se concederán contratos para la evaluación del centro médico de Belt Sahour en Palestina y para el apoyo psicosocial en 19 campos de refugiados de Cisjordania ${ }^{58}$.

Entre 2008 y 2010, en el caso de Palestina, a lo largo de estos años, también se solicitaría desde el Parlamento, la comparecencia de diversas personalidades palestinas para informar acerca de los territorios ocupados, las propuestas de resistencia popular y pacífica así como las actividades de los movimientos internacionales de solidaridad ${ }^{59}$. Además, en uno de los viajes oficiales a Israel, se estudiarían los avances para la cooperación entre ambos países, así como participarían en el encuentro de Donantes y Huéspedes de Agencia de las Naciones Unidas para la Ayuda a los Refugiados de Palestina en Oriente Próximo, y en la Mesa de Donantes de la Agencia de las Naciones Unidas para la Ayuda a los Refugiados de Palestina en el Próximo Oriente (UNRWA), fortaleciendo su compromiso con el pueblo palestino ${ }^{60}$. Del mismo modo que en el caso de Aminatu Haidar, el Parlament también reaccionaría frente al ataque, en 2010, del ejército israelí a la Flota de la Libertad formada por un convoy internacional de nueve barcos con ayuda humanitaria destinada a la Franja de Gaza ${ }^{61}$.

Entre 2011 y 2012, en cuanto a Palestina, tan solo consta una propuesta referente al reconocimiento del estado palestino y la aprobación de la Resolución 160/IX sobre el reforzamiento de las actuaciones de cooperación y paz en Palestina ${ }^{62}$. Un dato interesante

54 Res 66/VIII del Parlament de Catalunya, sobre el Plan director de cooperación al desenvolupament 2007-2010, Núm. exp.: 259-00001/08.

55 Res 754/VIII del Parlament de Catalunya, per la qual s'aprova el Pla director de cooperación al desenvolupament 2011-2014, Núm. exp.: 259-00003/08, 22 de juliol de 2010.

56 En 2007, el director de l'Agència Catalana de Cooperació al Desenvolupament, realitzaría un viatge de seguiment de projectes a Palestina. Butlletí oficial del Parlament, Legislatura VIII, Nº 331, 14 d'octubre de 2008.

57 Butlletí oficial del Parlament, Legislatura VIII, No 211 , 12 de febrer de 2008.

58 Butlletí oficial del Parlament, Legislatura VIII, Nº490, 19 de juny de 2009.

59 Butlletí oficial del Parlament, Legislatura VIII, N 537, 24 de novembre de 2008; Nº 369, 9 de desembre de 2008.

60 Butlletí Oficial del Parlament, Legislatura VIII, N³99, 10 de febrer de 2009.

61 Butlletí Oficial del Parlament, Legislatura VIII, No 732, 7 de juny de 2010. Declaració de la Junta de Portaveus del Parlament de Catalunya de condemna de l'atac de l'exèrcit d'Israel a la Flota per la Llibertat, Tra. 401-00050/08.

62 Res 160/IX del Parlament de Catalunya, sobre el reforçament de les actuacions de cooperació al desenvolupament i de construcció de la pau amb Palestina, Tram. 250-00257/09, Sessió núm. 4, 01.07.201 1, DSPC-C 112.
El hecho de comerciar con Israel y al mismo tiempo pedir la autodeteminación para Palestina, esjustificado por Catalunya como su estrategia para contribuir en el conflicto y la paz del Oriente Medio con una política de diálogo con las dos vertientes 
dentro de este periodo sería uno de los datos extraídos de la Propuesta de resolución sobre la oficialidad de las selecciones deportivas catalanas, en la que ERC, habla de "naciones o países que, todo y no disponer de Estado propio, pueden competir de igual a igual con federaciones de otros países como, [...] Palestina $[\ldots]^{\prime 63}$.

A partir de 2013, se redactará una declaración por parte de la Junta de portavoces del Parlament de Catalunya apoyando el fin de la ofensiva en Gaza y los ataques de grupos armados palestinos contra Israel. Como consecuencia de la intensificación del conflicto, se admitiría la Resolución 843/X sobre ayuda humanitaria en la Franja de Gaza ${ }^{64}$ y la Resolución 12/XI sobre el conflicto y la situación de los refugiados ${ }^{65}$.

El hecho de comerciar con Israel y al mismo tiempo pedir la autodeterminación para Palestina, es justificado por Catalunya como su estrategia para contribuir en el conflicto y la paz del Oriente Medio con una política de diálogo con las dos vertientes. Además, la Generalitat de Catalunya ha conseguido colaborar tanto a niveles administrativos como puede ser la Autoridad Nacional de Palestina o el Ayuntamiento de Gaza, y con agencias internacionales, como la UNRWA u ONG locales ${ }^{66}$. En el mismo caso, en 2014, BOC del 2 de julio de $2014^{67}$ (tram300/00188/10), se señalará la relación del Govern con Israel a partir de acuerdos y relaciones económicas y políticas, señalando que el presidente Mas había declarado que "Israel es un compañero de viaje claramente escogido por Cataluña”.

Por otro lado, cabe subrayar que, en 2013, tras la visita del expresidente Mas a Israel, se intensificarían los acuerdos y relaciones con el Gobierno de Israel. Mas, recientemente en 2015, catalogaría dicho país como el "compañero de viaje claramente elegido por Catalunya” y destacaría que los israelís "conocen muy bien qué es construir un Estado y luchar para preservar una lengua y una identidad”68, mientras que, en 2011, hablaría de Palestina, como un país que entienden del mismo modo que Catalunya, a los pueblos que desean vivir "en libertad, paz y prosperidad” 69 . La política de colaboración empresarial con empresas de seguridad ${ }^{70}$ Israel y Catalunya, así como de otro tipo de negocios o intereses como los llevados con Marruecos, refuerza y además legitima la ocupación y la violación sistemática de los derechos de ambos pueblos. Por esta razón, tras la visita al Estado israelí, Mas se reuniría con la comunidad palestina

63 Proposta de resolución sobre l'oficialitat de les seleccions esportives catalanes. Butlletí oficial del Parlament, Legislatura IX, Nº 325, 30 de maig de 2012.

64 Res 843/X del Parlament de Catalunya, d'ajut humanitari a la Franja de Gaza, Tram. 250-012223/10, Sessió núm. 19, 24.10.2014, DSPC-C 518.

65 Res 12/X del Parlament de Catalunya, sobre el conflicte entre Israel i Palestina i sobre la situació dels refugiats afectats pel conflicte bèl.lic a Síria, Tram. 250-00038/1 1, Sessió núm. 3, 25.02.2016, DSPC-C 49.

66 Butlletí, Op.Cit., 2005.

67 Butlletí, Op.Cit, 2014.

68 Sabé Mar. Mas convida Israel a covnertir-se en el company de viatge de Catalunya. El Món [en línea], Septembre de 2015. Disponible en http://elmon.cat/noticia/96926/index.html [Consultat el 28 de juliol de 2017].

69 President Mas: "Catalunya i Palestina entenen perfectament aquells pobles que desitgen viure en llibertat, pau i prosperitat", Generalitat de Catalunya, president.cat [en línea], Juliol 2011. Disponible en http://www.president. cat/pres_gov/AppJava/president/notespremsa/1 16791/president-mas-catalunya-palestina-entenen-perfectamentpobles-desitgen-viure-llibertat-pau-prosperitat.html [Consultat el 28/06/2017].

70 Como el envío de Agentes de los Mossos d'Esquadra quiénes han recibido instrucciones en Israel sobre técnicas de seguridad ciudadana. Bou, David, "Agents dels Mossos d'Esquadra reben instrucció a Israel sobre tècniques de seguretat ciutadana”, Directa [en línea], Febrer de 2014. Disponible en https://directa.cat/agents-dels-mossosdesquadra-reben-instrucci\%C3\%B3-israel-sobre-t\%C3\%A8cniques-de-seguretat-ciutadana [Consultado el 28/06/2017]. 
en Catalunya, para rebajar posibles tensiones provocadas por el viaje ${ }^{71}$. Muchos opinarán que el gobierno catalán está sometido bajo la influencia de Israel, como se puede observar en uno de los debates ocurridos en el Parlament como consecuencia de la prohibición de la entrada de banderas y pancartas palestinas a un partido de básquet en el que jugaban C. Barcelona y el Maccabi Tel Aviv ${ }^{72}$.

El último dato sobre las acciones dirigidas hacia Palestina es la creación en 2016 del Intergrupo de Palestina, pero sus actividades no entran en nuestro periodo de estudio ${ }^{73}$.

\section{El porqué de las diferencias entre los partidos políticos}

En julio de 2014 el Parlament rechazó el derecho de autodeterminación de palestinos, kurdos y saharauis presentado a través de una moción de ICV. ICV-IUiA se posicionarían a favor de Palestina, saharauis y kurdos, mientras que CiU y PPC estarían en contra. Por otro lado, el PSC votaría a favor de los palestinos y los saharauis mientras que Cs tan solo saharauis. No obstante, tras los debates y votaciones realizadas, no se produciría un consenso al respecto.

A partir de estos datos, nos preguntamos por qué existen estas diferencias entre partidos dependiendo de los pueblos a los que se dirija la iniciativa. No obstante, en el caso de Palestina, la cuestión podría plantear divergencias entre CDC (Convergència Demòcratica de Catalunya, actual PDeCAT) y ERC, ya que los primeros han demostrado en diversas ocasiones su afinidad o apoyo hacia Israel, como ya ha sido mencionado en el apartado de Palestina. También ocurriría en el caso del Sáhara y las relaciones del Govern con Marruecos.

En cuanto a $\mathrm{CiU}$ y PPC, se sitúan en contra del proceso de autodeterminación de los palestinos, el reconocimiento del derecho a la autodeterminación de los kurdos y el proceso de referéndum en el Sáhara occidental. En el caso del PPC está clara su posición ya que no bascula en ningún caso la idea del independentismo. No obstante, si analizamos a CiU, definido como un partido nacionalista catalán (que se disolverá en 2015), votarían en contra de un proceso que posteriormente ellos defenderían para Cataluña entre 2010 y 2015 con su apuesta independentista. Ahora bien, su posicionamiento fue argumentado explicando que ellos pedían el derecho a la autodeterminación de todos los pueblos, y no tan solo de estos tres.

Durante la interpelación al Govern sobre la política de acción exterior, Sara Vilà Galan, del Grupo Iniciativa Verds-Esquerra Unida i Alternativa, expondría que dicha moción es "una moción de todos" y no solo de su propio partido y reclamaría la desvinculación del Govern en las actividades y acuerdos relacionados en Israel, "o que por lo menos se expliquen con transparencia". Por otro lado, el uso de términos como "Israel es un apartheid como lo era Sudáfrica” no ayudaría a un consenso entre los parlamentarios. Así, Ferran Pedret i Santos, del PSC, haría referencia a la gran parte de conflictos que hoy en día se producen en el mundo concluyendo que hay que proteger a todos. Jordi Solé i Ferrando, de ERC, siguiendo en la línea
Enjulio de 2014 el Parlament rechazó

el derecho de autodeterminación de palestinos, kurdos y saharauis presentado a través de una moción de ICV

71 "Mas s'ha reunit amb la comunitat palestina a Catalunya després del viatge a Israel” El Punt Avui [en línea], desembre 2013. Disponible en: http://www.elpuntavui.cat/article/3-politica/17-politica/699150-mas-sha-reunitamb-la-comunitat-palestina-a-catalunya-despres-del-viatge-a-israel.html [Consultado el 28/06/2017].

72 Butlletí oficial del Parlament, Legislatura X, Nº522, 24 de març de 2015; preguntas planteadas en el $N^{\circ} 532,8$ d'abril de 2015; respuesta del gobierno en el No 568, 12 maig 2015.

73 Ver por ejemplo, Maite Gutiérrez, El Parlament analizará la situación de Palestina, La Vanguardia, 25/02/16. 
de Sara Vilà Galan, expondría que: "Nosotros entendemos que nuestro país [Cataluña] ha de tener vínculos institucionales, comerciales, universitarios, etcétera con Israel, pero también entendemos que no se pueden cruzar líneas rojas marcadas por la comunidad internacional", confirmando su posicionamiento hacia la mayoría de puntos de la moción dirigidos al derecho a la autodeterminación de dichos pueblos. En cuanto a José María Espejo-Saavedra Conesa, de Cs, recordará que Cataluña solo puede llevar a cabo acción exterior y no política exterior como está reconocido en el Estatuto de autonomía, acusando al Govern de querer "jugar a los estados" al intentar resolver estos conflictos sin pasar antes por las normativas del Gobierno central de España. Hace referencia al caso kurdo al exponer que, según dicha moción, se exige al Govern intervenir en el conflicto y no al Gobierno español. Además, votará a favor del pueblo saharaui, aunque para ellos el que deberá manejar la situación es el Gobierno central.

Por otro lado, Mireia Canals i Botines, de CiU, expondría la negativa de su partido frente a dicha moción al estar "dirigida a desestabilizar el gobierno de la Generalitat", aunque sí que reconocen el derecho a la autodeterminación y, al estar basada en un proyecto de ley sobre política exterior que no había sido todavía debatido. No obstante, argumentaría que "ni este Parlament ni el Govern de la Generalitat deben pretender resolver por nosotros todo aquello que representan los conflictos incluidos en esta moción”, además, argumenta que hoy en día lo que único que el Parlament puede hacer es una "contribución declarativa” porque a nivel internacional no tienen presencia ya que "lamentablemente" no son un Estado. En este caso, CiU se posicionará en contra ya que no están de acuerdo en la manera en la que ICV-EUiA quiere dirigir la política exterior de Cataluña, ya que tienen que pensar en promover una política "menos declarativa para quedar bien y más incisiva y proactiva para el futuro [... por lo que son y por lo que quieren llegar a ser”. Desde el PP, con Juan Milián Querol, acusarán a ICV-EUiA de defender los derechos de unos países y no de otros (como el caso de Venezuela) y de no respetar el trabajo del Intergrupo sobre el Sáhara Occidental, negándose por completo a aceptar la moción propuesta, al argumentar que sus propuestas son "hipócritas". El grupo mixto, por su parte, con Quim Arrufat Ibáñez, se posicionará favorable a tales medidas defendiendo el derecho a los pueblos a decidir.

En resumen, para el PP y Cs no existe la opción de comportarse como un Estado aplicando dichas mociones en un gobierno autonómico, mientras que para $\mathrm{CiU}$, todavía quedaba mucho que construir en la política exterior catalana para poder actuar de dicha manera. El PSC no estaría de acuerdo en que la propuesta se concentrase en dichos temas y no incluir más. No obstante, para partidos como ERC, ICV y el grupo mixto, por delante de todo está la defensa de los derechos principales de los ciudadanos y el derecho a decidir democráticamente.

\section{A modo de conclusión}

Para comprender el uso de la palabra autodeterminación en la estructura política catalana, hemos analizado discursos, debates y boletines del Parlament catalán. Además de que las propuestas realizadas por partidos han sido tratadas desde los diversos discursos políticos, a favor o en contra de la independencia, hay que tener en cuenta también el momento y las actividades exteriores que se han producido a lo largo de este periodo.

Tras este análisis, podemos concluir que nos encontramos ante un caso claro de protodiplomacia desde el año 2003 hasta la actualidad. Este cambio de paradiplomacia a protodiplomacia significa que hoy en día los partidos políticos catalanes han instrumentalizado otros reclamos de

\section{El nacionalismo} catalán ha expresado su sensibilización hacia las naciones sin Estado, así como al derecho a la autodeterminación, como en el caso del Sáhara occidental y Palestina, aunque también se posicionan hacia otros territorios como el kurdo 
autodeterminación dentro de los movimientos de independencia para promover, o al contrario limitar, cualquier demanda similar en Cataluña. Así este estudio contextualizado entre 2003 y 2016, muestra que existe un claro posicionamiento a favor de la independencia catalana, pero también una posición en contra que justifica la postura de todos los partidos políticos catalanes sobre las cuestiones de autodeterminación en el Sáhara Occidental y en Palestina.

Este artículo muestra que, a largo de estos años, el nacionalismo catalán ha expresado su sensibilización hacia las naciones sin Estado, así como al derecho a la autodeterminación, como en el caso del Sáhara occidental y Palestina, aunque también se posicionan hacia otros territorios como el kurdo. No obstante, a partir de este análisis se puede observar que además no existe un acuerdo entre los partidos políticos como consecuencia de sus diferencias sobre el estatuto deseado para la misma Cataluña.

Este trabajo es, hasta cierto punto, un pilot-study que abre la vía a más estudios sobre estos temas en el futuro. Tanto serán necesarias más aproximaciones cuantitativas como especialmente cualitativas.

\section{Bibliografía}

Aldecoa Luzárraga, F., \& Keating, M. (1999). Paradiplomacy in Action: The Foreign Relations of Subnational Governments. London: Cass.

Bayne, N., \& Woolcock, S. (2011). The New Economic Diplomacy Decision-Making and Negotiation in International Economic Relations. Farnham: Ashgate.

Bizoux, A. (2006). Catalogne: L'Émergence d'une Politique Extérieure. Strasbourg: Université Institut d'études politiques de Strasbourg.

Bursens, P., \& Högenauer, A-L. (2017). Regional parliaments in the EU multilevel parliamentary system. The Journal of Legislative Studies, 1-17.

Cofelice, A. (2012). Interregional Parliamentary Assemblies: A New Layer in the Multilevel Global Governance System. In L. Bekemans (Ed.), Intercultural Dialogue and Multilevel Governance in Europe: A Human Rights Based Approach (pp. 291-292). Brussels: P.I.E. Peter Lang.

Cofelice, A., \& Stavridis, S. (2017). Mapping the proliferation of parliamentary actors in the Mediterranean: facilitating or hindering cooperation? IAI Working Paper, Rome, April: http:// www.iai.it/en/pubblicazioni/mapping-proliferation-parliamentary-actors-mediterranean

Cornago, N. (2010). On the normalization of sub-state diplomacy. The Hague Journal of Diplomacy, 5(1-2), 11-36.

Costa, O., Dri, C., \& Stavridis S. (Eds.). (2013). Parliamentary Dimensions of Regionalization and Globalization. Basingstoke: Palgrave Macmillan.

Criekemans, D. (2010). Regional sub-state diplomacy from a comparative perspective: Quebec, Scotland, Bavaria, Catalonia. The Hague Journal of Diplomacy, 5(1-2), 37-64.

Crum, B., \& Fossum, J. E. (Eds.). (2013). Practices of interparliamentary coordination in international politics. Colchester: ECPR Press.

De Castro, J-L., y Ugalde Zubiri, A. (2004). La acción exterior del País Vasco (1980-2003). Bilbao: Instituto Vasco de Administración Pública. 
De Castro, J-L., y Ugalde Zubiri, A. (2011). Anuario sobre la acción exterior de Euskadi 2008 y 2009. Oñati: IVAP.

De Puig, L. M. (2008). International Parliaments. Strasbourg: Council of Europe Publishing.

Feliu, L., \& Serra, F. (2016). Catalan Parliamentary Diplomacy toward the Mediterranean. Mediterranean Quarterly, 27(4), 119-148.

García Pérez, R. (2009). La acción exterior de las Comunidades Autónomas en las reformas estatutarias. Madrid: Tecnos.

Hocking, B. (2011). Non-State Actors and the Transformation of Diplomacy. In B. Reinalda (Ed.), The Ashgate Research Companion to Non-State Actors (pp. 225-236). Farnham: Ashgate.

Huliaras, A., \& Tzifakis, N. (2010). Celebrity Activism in International Relations: In Search of a Framework for Analysis. Global Society, 24(2), 255-274.

Jancic, D. (2015). Globalizing Representative Democracy: The Emergence of Multilayered International Parliamentarism. Hastings International and Comparative Law Review, 38(2), 197-242.

Kissling C. (2011), The Legal and Political Status of International Parliamentary Institutions. Berlin: Committee for a Democratic U.N.

Kitsou, S. (2013). The Power of Culture in Diplomacy: The Case of U.S. Cultural Diplomacy in France and Germany. Exchange, 2(1). Retrieved from https://surface.syr.edu/exchange/ $\operatorname{vol} 2 /$ iss $1 / 3 /$

Melissen, J. (2005). The New Public Diplomacy: Soft Power in International Relations. Basingstoke: Palgrave Macmillan.

McHugh, J. T. (2015). Paradiplomacy, protodiplomacy and the foreign policy aspirations of Quebec and other Canadian provinces. Canadian Foreign Policy Journal, 21 (3), 238-256.

Noferini, A. (2016). El Govern de la Generalitat de Cataluña en la Unión Europea. La red de los actores. IEA-Recerca 2. Barcelona: Generalitat de Catalunya. Institut d'Estudis de l'Autogovern. Recuperado de http://www.gencat.cat/governacio/pub/sum/iea/ieaR_02.pdf

Oliveira Martins, B. (2015). "A Sense of Urgency": The EU, EU Member States and the Recognition of the Palestinian State. Mediterranean Politics, 20(2), 281-287.

Palermo, F., \& Kössler, K. (2017). Comparative Federalism: Constitutional Arrangements and Case Law. Oxford: Hart Publishing.

Paquin, S. (2004). Paradiplomatie et relations internationales: théorie des stratégies internationales des régions face à la mondialisation. Bruxelles: Presses Interuniversitaires Européennes.

Palomares Amat, M. (2011). La participación del parlamento de Cataluña en la aplicación y el control del principio de subsidiariedad. Revista de Derecho Comunitario Europeo, 38. Recuperado de http://www.catalunyaeuropa.net/img/pdf/Miquel_Palomares_2.pdf

Palomares Amat, M. (2016). La participació del Parlament de Catalunya en l'Assemblea Parlamentària de la Francofonia/ La participation du Parlement de Catalogne à l'Assemblée parlementaire de la Francophonie. Barcelona: Parlament de Catalunya, Testimonis Parlamentaris 37. Recuperado de http://apf.francophonie.org/IMG/pdf/la_participation_ du_parlement_de_catalogne_a_l_assemblee_parlementaire_de_la_francophonie._pdf 
Pasquier, R. (2012). Quand le local rencontre le global: contours et enjeux de l'action internationale des collectivités territoriales. Revue française d'administration publique, $141,167-182$.

Piazzi, S. (2015). Contribution by Ambassador Sergio Piazzi, Secretary General of the Parliamentary Assembly of the Mediterranean (PAM) on the occasion of the conference: South East European cooperation process parliamentary assembly - regional synergies, strategic cooperation and parliamentary diplomacy (Sofia, Bulgaria, 6 november 2015).

Reinalda, B. (Ed.). (2011). The Ashgate Research Companion to Non-State Actors. Farnham: Ashgate.

Sabic, Z. (2008). Building Democratic and Responsible Global Governance: The Role of International Parliamentary Institutions. Parliamentary Affairs, 61(2), 255-271.

Skoutaris, N. (2012). Comparing the Subnational Constitutional Space of the European SubState Entities in the Area of Foreign Affairs. Perspectives on Federalism, 4(2). Retrieved from http://www.onfederalism.eu/attachments/142_download.pdf

Stavridis, S. (2016). Conference sur "La diplomatie parlementaire dans l'espace méditerranéen: quel impact?". Séminaire régional de l'Organisation Internationale de la Francophonie sur "La diplomatie parlementaire: quel rôle pour les parlements dans les relations internationales?" Académie Diplomatique de Vienne, 13-17 Juin.

Stavridis, S., Gianniou, M., \& Cofelice, A. (2016). EU National Parliaments and the Recognition of Palestine: "Really" Breaking New Ground or "Just" Adding Further Support? Comillas Journal of International Relations, 6, 40-60.

Stavridis, S., \& Jancic, D. (Eds.). (2017). Parliamentary Diplomacy in European and Global Governance. Diplomatic Studies Series. Leiden/Boston: Brill Nijhoff.

Telò, M., Fawcett, L., \& Ponjaert, F. (Eds.). (2015). Interregionalism and the European UnionA Post-Revisionist Approach to Europe's Place in a Changing World. Ashgate.

The Hague Journal of Diplomacy (2013). Volume 8, Special Issue on "Sports Diplomacy".

Warleigh, A., Robinson, N., \& Rosamond, B. (Eds.). (2013). New Regionalism and the European Union - Dialogues, comparisons and new research directions. London: Routledge/ECPR Studies in European Political Science.

Watson, A. (1982). Diplomacy - The Dialogue Between States. London: Methuen.

Weisglas, F., \& de Boer, G. (2007). Parliamentary Diplomacy. The Hague Journal of Diplomacy, 2(1), 93-99.

Wolff, S. (2007). Paradiplomacy. Scope, Opportunities and Challenges. The SAIS Europe Journal. Retrieved from http://www.saisjournal.org/posts/paradiplomacy 